\title{
Atrophy and High Intensity Lesions: Complementary Neurobiological Mechanisms in Late-Life Major Depression
}

\author{
Anand Kumar, M.D., Warren Bilker, Ph.D., Zhisong Jin, M.S., and Jayaram Udupa, Ph.D.
}

\begin{abstract}
The primary objective of our study was to examine the role of atrophy, high intensity lesions and medical comorbidity in the pathophysiology of major depressive disorder in the elderly (late-life MDD). Our sample was comprised of 51 patients with late-life MDD and 30 non-depressed controls. All subjects were scanned on 1.5 tesla magnetic resonance imaging scanner (MRI) and absolute and normalized measures of brain and lesion volumes were obtained and used for comparison between groups. Patients with MDD had significantly smaller frontal lobe volumes, together with larger whole brain lesion volumes when compared with controls $(\mathrm{p}<.05)$. Whole brain lesion volumes correlated significantly $(\mathrm{r}=0.41, \mathrm{p}=.006)$ with overall medical comorbidity. The odds ratio (OR) for existing $M D D$ increases significantly with a decrease in frontal lobe
\end{abstract}

volume and an increase in whole brain lesion volumes $(\mathrm{p}<$ .05). Our findings suggest that atrophy and high intensity lesions represent relatively independent pathways to latelife MDD. While medical disorders lead to neuropathological changes that are captured on $M R$ imaging as high intensity signals, atrophy may represent a relatively autonomous phenomenon. These findings have broad implications for the pathophysiology of mood disorders and suggest that complementary neurobiological processes may lead to cumulative neuronal injury thereby predisposing to clinical depression.

[Neuropsychopharmacology 22:264-274, 2000] (C) 2000 American College of Neuropsychopharmacology. Published by Elsevier Science Inc.
KEY WORDS: Late-life depression; Atrophy; Major depression; Magnetic resonance imaging; Geriatric depression; High intensity lesions; Medical comorbidity

Depression is one of the most common mental disorders in the elderly (Alexopolous et al. 1988; Beck and Koenig 1996; Blazer et al. 1987; Blazer 1989, 1994; Koenig et al. 1993; Oxman et al. 1987; Parmalee et al.

From the UCLA-Neuropsychiatric Institute (AK, ZJ), Los Angeles, CA; Neuropsychology (WB), University of Pennsylvania, Philadelphia, PA; and Department of Radiology (JU), University of Pennsylvania, Philadelphia, PA.

Address correspondence to: Anand Kumar, MD, UCLA-Neuropsychiatric Institute, 37-384B, 760 Westwood Plaza, Los Angeles, CA 90024, Tel.: (310) 206-4405; Fax: (310) 794-9011; E-mail: akumar@ mednet.ucla.edu

Received April 26, 1999; revised August 25, 1999; accepted September 17, 1999.
1989; Ruegg et al. 1988; Sherbourne et al. 1994). The prevalence of major depressive disorder (MDD) is estimated to be approximately 1-2 percent in community settings and strikingly higher in inpatient and long term care settings (Blazer et al. 1987; Koenig et al. 1993; Parmalee et al. 1989). The prevalence of other clinically significant forms of depression, that do not meet the severity criteria for MDD, lies between 5 and 15 percent in all major clinical settings (Beck and Koenig 1996; Blazer et al. 1987, Blazer 1994; Parmalee et al. 1989). Anatomical and physiological neuroimaging approaches have been utilized to study the neurobiological correlates of MDD and minor depression in the elderly (Coffey et al. 1990; Coffey et al. 1993; Greenwald et al. 1996; Krishnan et al. 1988; Krishnan 1993; Kumar et al. 1993; Kumar et al. 1997a; Lesser et al. 1994; Sackheim et al. 1993; Sheline et al. 1996). Glucose hypometabolism and 
reductions in blood flow have been demonstrated in neocortical and subcortical regions in elderly patients with MDD when compared with non-depressed controls using positron emission tomography (PET), single photon emission computed tomography (SPECT) and Xenon 133 inhalation techniques (Kumar et al. 1993; Lesser et al. 1994; Sackheim et al. 1993). Using magnetic resonance imaging (MRI), investigators have demonstrated neuroanatomical abnormalities including smaller focal brain volumes and larger high intensity lesion volumes in patients with both late-life major and minor depression when compared with non-depressed controls (Coffey et al. 1990; Coffey et al. 1993; Greenwald et al. 1996; Krishnan et al. 1988; Krishnan 1993; Kumar et al. 1997a, 1997b, 1998; Sheline et al. 1996).

Despite these observations, the precise nature and extent of the neuroanatomical changes in elderly patients with MDD remains unresolved (Jeste et al. 1988; Morris and Rapoport 1990).

Late-life MDD is also consistently associated with medical comorbidity (Berkman et al 1986; Gierz and Jeste 1993; Katz et al. 1994; Katz 1996; Lacro and Jeste 1994; Rodin and Voshart 1986). A broad spectrum of medical disorders including cardiovascular, musculoskeletal, gastrointestinal, pulmonary and metabolic disturbances are associated with both major and minor forms of depression (Alexopolous et al. 1988; Katz et al. 1994; Koenig et al. 1993; Lacro and Jeste 1994). Despite this association, the relationship between neuroanatomical abnormalities and medical disorders in late-life and their relative contributions to the pathophysiology of mood disorders remain unknown.

In an earlier report, we demonstrated that the odds of developing MDD in late-life increased with overall medical burden and larger whole brain cerebrospinal fluid (CSF) volume (Kumar et al. 1997a). However, only global measures of whole brain CSF and ventricular volumes were obtained in that study and these served as indirect measures of brain atrophy. In addition, estimates of high intensity lesions that were used in our analyses were qualitative in nature. Consequently, no statement was made about regional brain volume differences between groups and the role of more precise quantitative estimates of atrophy and high intensity lesion volumes in the development of MDD.

The principal objective of our current study was to examine the relative roles of atrophy, high intensity lesions and medical disorders in the development of MDD in the elderly. We were further interested in clarifying the relationship between quantitative neuroanatomic measures and objective clinical indices such as specific cerebrovascular risk factors and reliable measures of overall medical burden. Such an approach, we believe, may provide us with insights into the mechanisms/pathways that may be involved in the pathophysiology of late-life MDD.

\section{SUBJECTS}

Our study groups were comprised of 51 patients with late-life Unipolar MDD diagnosed using DSM IV criteria (American Psychiatric Association 1994) and 30 non-depressed controls (Table 1). Twenty-eight of our current sample of 51 patients with MDD have been previously reported on (Kumar et al. 1997a). The controls used in this study also served as the control group in our recent MRI report on minor depression (Kumar et al 1997b) but were not part of our earlier MDD study (Kumar et al. 1997a). In order to be included in the study, the MDD patients had to meet the following:

\section{Inclusion Criteria:}

1. DSM IV criteria for MDD;

2. A score of 15 or greater on the 17 item Hamilton Depression Rating Scale (Hamilton 1967);

3. Mini Mental State Exam (MMSE) Scores of 24 or greater (Folstein et al 1975).

\section{Exclusion Criteria:}

1. Clinical evidence of any other brain disorder such as stroke, transient ischemic attacks (TIA) or Parkinson's disease;

2. History and/or mental status exam suggestive of a progressive dementia;

3. MMSE score less than 24;

4. History of substance abuse.

\section{Entry criteria for controls:}

1. Age 60 or greater;

2. Free of current and past psychopathology including substance abuse;

3. No evidence of any brain disease such as stroke, TIA or dementia.

Psychopathology was ruled out on the basis of mental status exams and the use of the structured interview (SCID for normals) based on the diagnostic and statistical manual for psychiatric disorders-DSM IV. Patients diagnosed with depression also received a DSM based structured psychiatric interview (SCID) in addition to the standard clinical psychiatric assessment.

MDD patients were recruited from the geriatric psychiatry inpatient and ambulatory care programs connected with the Hospital of the University of Pennsylvania. After complete description of the study to the subjects, written informed consent was obtained whenever appropriate. The geropsychiatry ambulatory care program at the University of Pennsylvania is a collaborative effort with the division of geriatric medicine and combines characteristics of a primary care and tertiary referral center. Controls were recruited from the community through newspaper advertisements.

Patients and controls had several stable comorbid medical disorders including ischemic heart diseases, diabetes and arthritis. Several patients with MDD were 
Table 1. Some of the Primary Demographic and Clinical Variables in and the Absolute and Normalized Brain and Lesion Volumes in Two Groups

\begin{tabular}{|c|c|c|c|c|}
\hline Index & $\begin{array}{c}\text { MDD } \\
\mathrm{n}=51 \\
\text { Gender: } 36 \mathrm{~F} \\
15 \mathrm{M} \\
\text { Age: } 74.3 \\
(6.56)\end{array}$ & $\begin{array}{c}\text { Controls } \\
n=30 \\
\text { Gender: } 23 \mathrm{~F} \\
7 \mathrm{M} \\
\text { Age: } 69.43 \\
(6.09)\end{array}$ & $\begin{array}{c}p \\
\text { Adjusting for } \\
\text { Age-Gender and ICV }\end{array}$ & $\begin{array}{c}p \\
\text { with CIRS } \\
\text { tot Adjustment }\end{array}$ \\
\hline MMSE & $\begin{array}{l}27.4 \\
(2.53)\end{array}$ & $\begin{array}{l}29.54 \\
(0.69)\end{array}$ & $\mathrm{N} / \mathrm{A}$ & $\mathrm{N} / \mathrm{A}$ \\
\hline CIRS & $\begin{array}{c}3.98 \\
3.98 \\
(3.39)\end{array}$ & $\begin{array}{c}(.07) \\
2.29 \\
(1.44)\end{array}$ & $\mathrm{N} / \mathrm{A}$ & $\mathrm{N} / \mathrm{A}$ \\
\hline CVRF & $\begin{array}{l}13.0 \\
(4.24)\end{array}$ & $\begin{array}{l}10.11 \\
(4.26)\end{array}$ & $\mathrm{N} / \mathrm{A}$ & $\mathrm{N} / \mathrm{A}$ \\
\hline Ham D & $\begin{array}{l}19.8 \\
(3.91)\end{array}$ & N/A & $\mathrm{N} / \mathrm{A}$ & $\mathrm{N} / \mathrm{A}$ \\
\hline Total Brain Volume(cc) & $\begin{array}{l}942.00 \\
(127.4)\end{array}$ & $\begin{array}{l}1008.65 \\
(128.69)\end{array}$ & $\mathrm{N} / \mathrm{A}$ & $\mathrm{N} / \mathrm{A}$ \\
\hline Total Brain/CV & $\begin{array}{c}0.834 \\
(0.0410)\end{array}$ & $\begin{array}{c}0.854 \\
(0.0617)\end{array}$ & .930 & .876 \\
\hline Frontal Brain & $\begin{array}{l}168.03 \\
(30.80)\end{array}$ & $\begin{array}{l}197.51 \\
(31.09)\end{array}$ & $\mathrm{N} / \mathrm{A}$ & $\mathrm{N} / \mathrm{A}$ \\
\hline Frontal Brain/CV & $\begin{array}{c}0.148 \\
(0.0173)\end{array}$ & $\begin{array}{l}0.167 \\
(0.0162)\end{array}$ & .0002 & .0002 \\
\hline Temporal Brain & $\begin{array}{l}176.55 \\
(29.65)\end{array}$ & $\begin{array}{l}202.5 \\
(35.1)\end{array}$ & $\mathrm{N} / \mathrm{A}$ & $\mathrm{N} / \mathrm{A}$ \\
\hline Temporal Brain/CV & $\begin{array}{c}0.156 \\
(0.0197)\end{array}$ & $\begin{array}{c}0.172 \\
(0.0266)\end{array}$ & .0943 & .1102 \\
\hline Lesion Volume(voxels) & $\begin{array}{c}1047.6 \\
(1523.54)\end{array}$ & $\begin{array}{c}122.00 \\
(184.38)\end{array}$ & .0349 & .0911 \\
\hline Lesion/CV & $\begin{array}{c}0.0033 \\
(0.0046)\end{array}$ & $\begin{array}{c}0.00037 \\
(0.00054)\end{array}$ & .0241 & .070 \\
\hline
\end{tabular}

The $p$ age-gender columns represent the significance level after adjusting for age and sex differences.

$p$ CIRS TOT is the $p$ value after additionally controlling for overall medical comorbidity.

$\mathrm{CV}=$ Total intracranial volume; MMSE = Mini Mental State Exam Scores; CIRS = Total Cumulative Illness Rating Scale Score; $\mathrm{CVRF}=\mathrm{Cere}-$ brovascular Risk Factor Score; HAMD = Score on the 17 item Hamilton Depression scale; N/A = not applicable.

on benzodiazepines and antidepressants at the time of the study. None of these medications were considered to contribute to the mood disorder. Thirty-five of the 51 patients with MDD had their first onset of depression at or later than age 60. The Cumulative Illness Rating Scale (CIRS) was used to quantify overall medical burden in both groups (Linn et al. 1968). The CIRS is a validated clinical instrument that categorizes disorders into 12 organ systems (gastrointestinal, cardiovascular etc.) and rates each of them along a 0-4 severity scale $(0=$ no illness, $4=$ severe illness). The CIRS is commonly used in geriatric research to obtain a quantified estimate of overall medical burden. The American Heart Association Scale for stroke risk factors was used to quantify cerebrovascular risk factors (American Heart Association 1990; Wolf et al. 1991). This scale incorporates eight independent measures; age, presence and treatment of hypertension, diabetes, smoking, coronary vascular disease, atrial fibrillation and left ventricular hypertrophy and provides a score indicating overall risk for cerebrovascular disease. CIRS and the stroke risk factor scales were administered by board-certified psychiatrists who were supervised by a geriatric psychiatrist
(AK) familiar with the use of these instruments. While both of our study groups had several comorbid medical disorders, the MDD group presented with greater overall medical burden. The MDD group was older than the control group though there was considerable overlap in age between subjects in both groups. The gender distribution was comparable in both groups.

\section{MRI METHODS}

MRI scans were obtained on a 1.5 tesla GE scanner with head coil in planes parallel to the canthomeatal line. Both $\mathrm{T} 2$ and proton density weighted images were obtained in all subjects (TR $=3000, \mathrm{TE}=30$ and $80 \mathrm{msec}$ ) and the images were displayed on a 256x256 matrix, with pixel size of $0.86 \mathrm{~mm}$ and field of view of $22 \mathrm{~cm}$ (Kohn et al. 1991; Kumar et al. 1994). The size of the individual voxels was $0.37 \mathrm{~mm}$ cubed and about 275 voxels approximated $1 \mathrm{cc}$ in volume. Axial slices were $5 \mathrm{~mm}$ thick and contiguous. A segmentation program developed within the Department of Radiology at the University of Pennsylvania and previously used to ex- 
amine neuroanatomical changes in subjects with major depression, schizophrenia and dementia of the Alzheimer type (DAT) was used in our image analysis (Kohn et al. 1991; Kumar et al. 1994). The temporal lobe outlined in our analysis included both lateral and mesial temporal structures and the frontal cortex did not include the sensorimotor region (Cowell et al. 1994). Details of the technique used to segment brain from cerebrospinal fluid (CSF) and the anatomic boundaries and landmarks used to delineate the frontal and temporal lobes have been described previously (Cowell et al. 1994, See Appendix). Manual tracing of all the neuroanatomical boundaries was done by one rater (ZJ) who was blind to the clinical status of all subjects. Good inter-rater reliability for all regions (intraclass correlation coefficient 0.90) was established between this rater and others who apply identical methods of regional neuroanatomical analyses to the study of neuropsychiatric disorders (Cowell et al. 1994; Kumar et al. 1994). Quantitative measures of total brain and high intensity lesion volumes were obtained together with estimates of frontal and temporal lobe volumes. Absolute brain and lesion volumes were additionally normalized to the intracranial volume (brain plus CSF) to correct for individual differences in head size.

\section{FUZZY VOXEL APPROACH}

To detect and quantify the volume of high intensity signals, a novel method of object delineation based on "fuzzy connectedness" was utilized (Udupa 1994; Udupa et al. 1994a; Udupa and Samarasekera 1996). An object such as a high intensity signal lesion is considered to be a fuzzily connected entity wherein every pair of voxels has a "strength of connectivity" associated with it utilized (Udupa 1994; Udupa et al. 1994a; Udupa and Samarasekera 1996). This strength is a global property which determines how voxels hang together in a fuzzy way to form an object (Figure 1). The power of this method compared to other segmentation methods comes from the consideration of an object as fuzzily defined as well as a fuzzily connected entity. A software system called 3DVIEWNIX in which this method was implemented was utilized in all image processing. This method requires two essential steps: In the first stage, minimal operator interaction is required and the signals are automatically detected by the computer (Udupa et al. 1994a; Udupa and Samarasekera 1996). A few false positives may be included at this stage. In the second stage, the operator accepts/rejects individual high intensity signals with the click of a mouse button, in response to the systems display of each 3-dimensional lesion. The decision to accept/reject individual lesions was made by a board-certified geriatric psychiatrist (AK) experienced in neuroanatomical imaging. The fuzzy connectedness approach eventually provides whole brain high intensity signal volume in absolute voxel numbers that can be converted to cubic centimeters and normalized to total intracranial volume. This system has been tested extensively and can be used

\section{A}

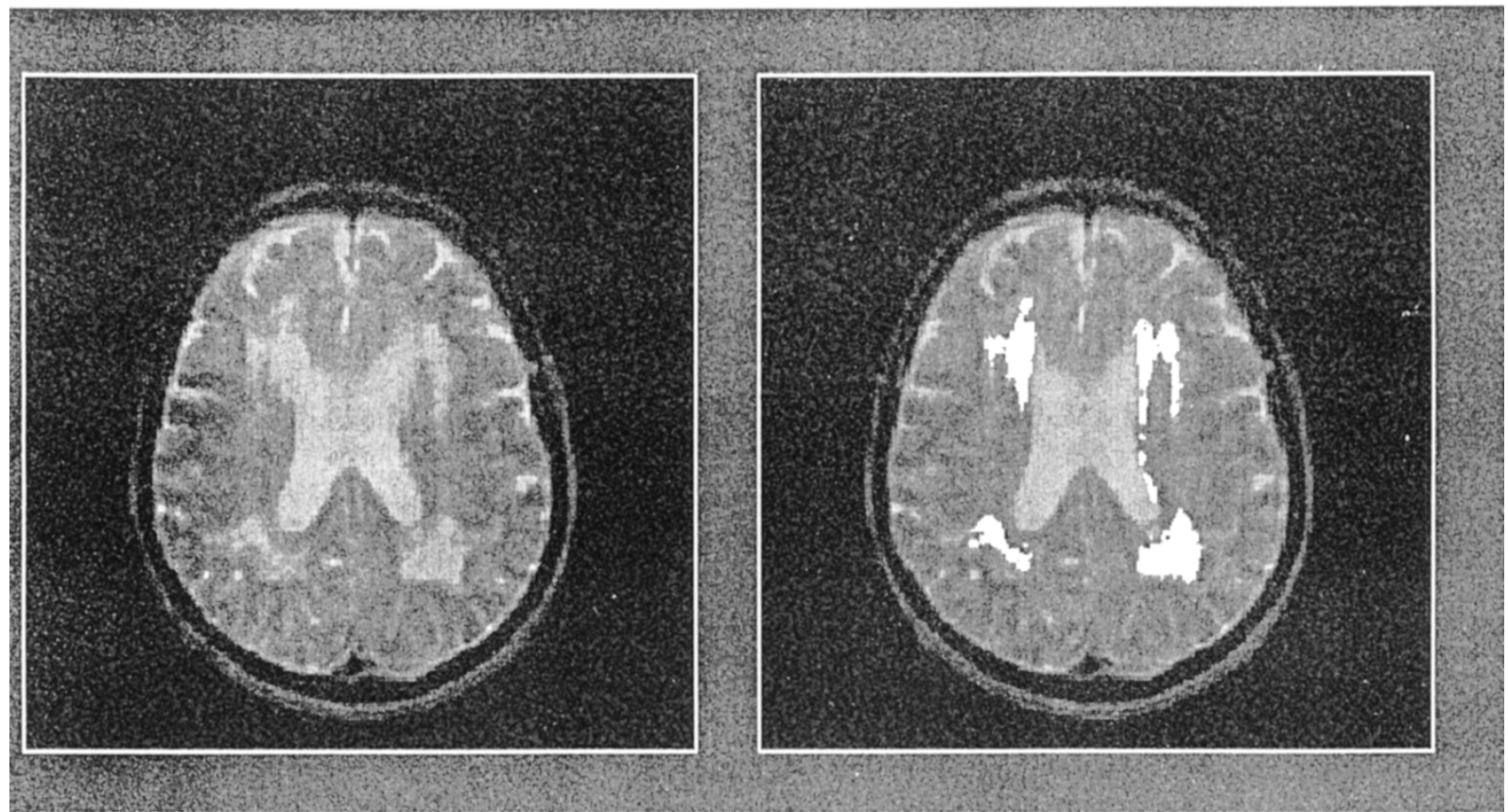

Figure 1. The left image shows high intensity lesions as they appear on a proton density image. The image on the right shows the same lesion after the fuzzy voxel lesion detection methodis applied. 
with good inter-rater reliability (intraclass correlation coefficients of 0.80 ).

\section{STATISTICS}

A linear regression was used to compare absolute and normalized measures of brain and lesion volumes between the two groups after controlling for the potential effects of age and gender. In a secondary analysis, we also introduced total CIRS scores as an additional covariate in our analysis in order to examine the influence of overall medical comorbidity, if any, on potential differences in brain and/or lesion volume between the two groups. A logistic regression was used to determine the influence of individual measures such as brain volume, lesion volume, overall medical burden and cerebrovascular risk factors on the odds ratio for the prevalence of existing MDD. The odds ratio from a logistic regression relating prediction to the prevalence of existing MDD, is sometimes referred to as the prevalence odds ratio (Seigel and Greenhouse 1973) (OR for MDD). The logistic regression was done in two steps: we first estimated the effects of each of these measures in increasing the OR for MDD (while controlling for intracranial volume in the case of brain and lesion volumes). In the next stage, we estimated the effects of the same measures while covarying for the other three variables. This provides us with an estimate of the ability of each of these measures to independently influence the odds for MDD. A partial Spearman correlation coefficient (after controlling for age) was used to examine the relationships between the various neuroanatomical and clinical indices of medical and cerebrovascular risk.

\section{RESULTS}

Our findings (Table 1) demonstrate that patients with MDD have significantly smaller normalized frontal lobe volumes when compared with the non-depressed controls after controlling for age and gender differences between the two groups. Normalized temporal lobe and whole brain volumes were also smaller in the depressed group, though the differences did not reach statistical significance. Though we present absolute and normalized measures of frontal and temporal lobe volumes for both groups (Table 1), statistical analyses for regional measures were performed only on normalized volumes. Absolute (actual number of voxels) and normalized measures of whole brain high intensity lesion volumes were significantly larger in the MDD group when compared with the controls $(p=0.034$ and 0.024 respectively). The statistically significant decrease in normalized frontal volume between groups remained after we adjusted for overall medical comorbidity, in addition to the existing age and gender controls. However, the differences in lesion volumes between the MDD and control groups were no longer statistically significant after adjusting for overall medical burden.

A logistic regression (Table 2) revealed that the OR for MDD increased with a decrease in frontal lobe volume and an increase in whole brain lesion volume. Table 2 presents the OR for MDD (with $95 \%$ confidence intervals) for each of our primary variables of interest both before (Unadjusted OR) and after covarying for the other three variables (Adjusted OR). A decrease in frontal lobe volume by $25 \mathrm{cc}$ (which approximates the mean difference between groups) increased the OR by 3.088 , while a frontal lobe volume decrease of $50 \mathrm{cc}$ increased the OR by 9.536 . The influence of a smaller frontal lobe volume on the OR for MDD persisted after controlling for the other three variables (Table 2).

An increase in lesion volume (measured in voxels) also significantly increased OR for MDD. An increase in lesion volume of 200 voxels increased the OR by 1.904 . Controlling for the other variables had an impact, albeit small, on the influence of lesion volumes on MDD - the statistical significance became insignificant ( $p$ value changed from 0.028 to 0.111 ), and the effect size decreased marginally after the introduction of these covariates. In order to further determine if smaller frontal lobe volumes and larger lesion volumes impacted on each other's ability to increase the OR for MDD, we estimated their individual impact on the OR while controlling for the other variable in a subanalysis. Smaller frontal lobe volumes and larger lesion volumes did not influence the ability of the other to increase the odds for depression. An increase in overall medical comorbidity (CIRS Score by a factor of 1) and cerebrovascular risk factors did not affect the OR for MDD in our sample

Table 2. The Odds Ratio for MDD with Incremental Decreases in Brain Volume and Increases in Whole Brain Lesion Volumes

\begin{tabular}{lcc}
\hline Variable & Unadjusted OR & Adjusted OR \\
\hline Frontal Brain & $p=.0087$ & $p=.0245$ \\
$25 \mathrm{cc}$ & $3.088(1.330-7.173)$ & $3.295(1.166-9.312)$ \\
$50 \mathrm{cc}$ & $9.536(1.767-51.450)$ & $10.859(1.386-86.713)$ \\
Lesion Vox & $p=.0283$ & $p=.1117$ \\
1 Vox & $1.0032(1.0003-1.0061)$ & $1.00026(0.999-1.0057)$ \\
200 Voxels & $1.904(1.0701-3.388)$ & $1.667(0.888-3.137)$ \\
CIRS & $p=.0963$ & $p=.5686$ \\
& $1.258(0.960-1.647)$ & $1.1213(0.756-1.662)$ \\
CVRF & $p=.1512$ & $p=.860$ \\
& $1.0370(0.864-1.245)$ & $0.979(0.773-1.240)$ \\
\hline
\end{tabular}

UNADJUSTED OR $=$ OR For MDD before controlling for other variables; ADJUSTED OR = OR for MDD after controlling for the other 3 variables; $\mathrm{CIRS}=$ Total Cumulative Illness Rating Scale Score; $\mathrm{CVRF}=$ Cerebrovascular Risk Factor Score. 
(Table 2). To additionally clarify the relative impact of medical comorbidity and lesion volume on MDD, we also examined the influence of each measure on the OR while controlling for the other. Neither measure significantly increased the OR for MDD while controlling for the other.

There is a significant correlation (Table 3) between total lesion volume and overall medical comorbidity $(r=$ $0.414, p=.0058$ ). The relationship between lesion volume and cerebrovascular risk factors only approached statistical significance $(r=0.283, p=.066)$. Frontal lobe volume did not correlate significantly with either overall medical comorbidity or cerebrovascular risk factors. There was also no significant correlation between frontal lobe volume and estimates of whole brain lesion volume. The two primary clinical measures of medical illness, the CVRF and the CIRS were significantly correlated $(r=0.34, p=0.025)$ in our study.

\section{DISCUSSION}

The principal finding to emerge from our study is that the odds for MDD increase significantly with a decrease in frontal lobe volume and an increase in whole brain high intensity lesion volume. Our data also suggest that atrophy and high intensity lesions may represent complementary pathways to late-life MDD in our sample: In our analyses, controlling for either variable did not impact on the ability of the other to increase the odds of developing major depression. Also, while lesion volumes are significantly correlated with overall medical burden, atrophy appears to be a relatively independent phenomenon that contributes to late-life MDD. While the MDD group also presented with smaller normalized temporal lobe and total brain volumes when compared with the control group, these differences were not statistically significant.

The frontal lobes have been implicated in a variety of behavioral and cognitive disorders including schizophrenia, obsessive compulsive disorder and frontotemporal dementia (Baxter et al. 1987; Fuster 1996; Kumar and Gottlieb 1993; Mishkin 1964; Weinberger et al.

Table 3. Spearman (Partial) Correlation Matrix, Adjusted For Age and ICV, Demonstrating the Relationships between the Primary Neuroanatomical and Clinical Measures

\begin{tabular}{llll}
\hline Index & CIRS Total & CVRF Total & Lesion Voxels \\
\hline Frontal & $r=0.161$ & $r=0.0174$ & $r=0.0385$ \\
$\quad$ Brain (cc) & $p=.304$ & $p=.912$ & $p=.807$ \\
Lesions & $r=0.414$ & $r=0.283$ & 1 \\
$\quad$ (Voxels) & $p=.0058$ & $p=.0657$ & \\
CVRF & $r=0.341$ & 1 & $r=0.283$ \\
& $p=.0252$ & & $p=.0657$ \\
\hline
\end{tabular}

1992). The frontal lobes occupy approximately one third of the cerebral cortex and the frontal cortex is one of the last regions of the cerebral cortex to develop both phylogenetically and ontogenetically. Both anatomically and histologically the frontal lobe is heterogeneous and the physiological functions attributed to this region reflect this heterogeneity (Fuster 1989; Fuster 1996; Kumar and Gottlieb 1993; Mishkin 1964). The frontal cortex has rich afferent and efferent connections to several limbic, subcortical and other neocortical regions (Stuss and Benson 1984). Evidence from primate and human studies indicate an important role for this region in the regulation of affect and in modulating certain cognitive functions consistent with its extensive neural connections (Fuster 1989; Fuster 1996; Mishkin 1964). In vivo neuroimaging studies corroborate preclinical observations and abnormalities in frontal anatomy and physiology have been demonstrated in several psychiatric disorders (Baxter et al. 1987; Fuster 1996; Mishkin 1964; Weinberger et al. 1992). Our findings extend these earlier observations and indicate that in addition to being smaller in patients with late-life MDD, frontal atrophy may also represent a relatively distinct pathway that compromises neural circuits and leads to MDD.

Atrophy in mood disorders may not however be restricted to the frontal region and the magnitude and extent of atrophy in MDD samples may vary depending on a number of underlying neurobiological factors (Caine et al. 1993; Coffey et al. 1993; Rabins et al. 1991; Sheline et al. 1996). Reports range from those that demonstrate a reduction in total brain volumes to others that indicate a more selective involvement in patients with MDD when compared with controls (Rabins et al. 1991; Sheline et al. 1996, Coffey et al. 1993). There are regional variations in the sensitivity of neurons to different biological insults. Hippocampal neurons are believed to be more cortisol sensitive while subcortical nuclei are well established sites of small "lacunar" infarcts in patients with long-standing vascular disease (Hastak and Hachinski 1992; Sapolsky and Pulsinelli 1985; Sapolsky et al. 1986, 1990; Schmidt et al. 1991). Hippocampal atrophy in depression may therefore be a reflection of hypercortisolemia that occurs with hypothalamic pituitary adrenal (HPA) axis dysfunction commonly observed in mood disorders (Sheline et al. 1996). While the precise causes of atrophy in patients with MDD are unknown, several neurobiological factors may be involved in this process. Reductions in the level of neurotrophins such as nerve growth factor (NGF) and alterations in molecules such as cytokines may also contribute to the atrophy observed in patients with MDD (Duman et al. 1997; Katz et al. 1994). Changes in the levels of cortisol and trophic factors are perhaps better conceptualized as an integral part of the perturbations of stress mediated pathways that are commonly encountered in depression (Duman et al. 1997; Sheline 
et al. 1996). The cumulative impact of these different neurobiological injuries on the brain would be to cause atrophy in sensitive regions that could collectively result in a reduction in total brain volume. The apparent discrepancy in reports of atrophy patterns in patients withMDD may more accurately reflect variability in the underlying biological processes that are selectively captured on MR imaging of specific study samples (Coffey et al. 1993; Kumar et al. 1997a; Lyness et al. 1998; Ruegg et al. 1988; Sheline et al. 1996).

The relationship between medical disorders, high intensity lesions and atrophy is complex and needs to be better elucidated. High intensity lesions occur predominantly in white matter regions of the brain and they represent a spectrum of pathological changes including ischemia, demyelination and edema (Boyko et al. 1994; Drayer 1988). Results from the Cardiovascular Health Study indicate that the frequent correlates of these lesions include age, silent stroke, hypertension and $\mathrm{FEV}_{1}$ (Longstreth et al. 1996). Other, as yet unidentified, biologic factors may also contribute to the MR signal (Ylikoski et al. 1995). In our current study, covarying for medical comorbidity eliminated the statistical significance in lesion volume, but not frontal lobe volume differences between the MDD and control groups. These findings, together with our data showing that lesion volumes correlate with measures of medical comorbidity and cerebrovascular risk factors, suggest that high intensity lesions are an integral part of the medical/ vascular complex frequently observed in elderly clinical populations. In our logistic regression, controlling for overall medical comorbidity did not appreciably impact on the ability of lesions to increase the OR for depression. We interpret this finding to indicate that despite the correlations between lesions and comorbidity, other biological factors, in addition to medical illness, probably contribute to the lesions and their impact on brain structure and function. In our MDD sample, atrophy appears to be independent of both overall medical burden and cerebrovascular risk factors. However, chronic ischemic changes have been shown to lead to atrophy, predominantly in the white matter, resulting in well recognized clinical/cognitive disturbances (DeReuck et al. 1980; Fisher 1989; Huang et al. 1985; Loizou et al. 1981). The structure of subcortical nuclei may also be influenced by chronic hypertension and lacunar infarcts (Schmidt et al. 1991; Hastak and Hachinski 1992). Ischemic neurons may in turn be more sensitive to stressors including psychological ones (Duman et al. 1997). Therefore, at a more basic cellular level, atrophic and ischemic changes may interact and amplify their individual impact on brain structure and function (Duman et al. 1997).

Late-life MDD is associated with several acute and chronic medical conditions involving multiple organ systems. Acknowledging this robust association, the $\mathrm{NIH}$ consensus statement on the diagnosis and treatment of late-life depression states "the hallmark of de- pression in late-life is its association with medical comorbidity" (NIH Consensus Panel 1992). Each disorder may lead to clinical depression via a distinct set of mechanisms such as vascular, immunologic, etc. The relative contribution of these disorders to mood disturbances is likely to vary substantially in different patient groups. While vascular mechanisms may be presumed in depression following acute vascular events such as myocardial infarction (post MI depression) and cerebrovascular accidents (post stroke depression), the mechanisms responsible for mood disturbances in more stable, chronically ill populations are likely to be multifactorial (Frasure-Smith et al. 1995; Glassman and Shapiro 1998; Rabins et al. 1985; Robinson et al. 1984). The apparent relationship between vascular disease and high intensity lesions on MR images of patients with MDD, has led investigators to suggest that vascular mechanisms may be the principal ones involved in latelife MDD (Krishnan et al. 1997; Alexopolous et al. 1997). While it may be possible to identify subgroups with clinical characteristics suggestive of a vascular etiology even in general settings, a conservative interpretation of findings is warranted when study samples are recruited from heterogeneous settings where vascular disease coexists with other medical disorders. Our demonstration of a statistically significant correlation between measures of overall medical and vascular burden in our sample further corroborates our contention that in primary care/ambulatory settings, vascular disease is perhaps better conceptualized as an important part of the total comorbidity picture, than as an isolated set of mechanisms.

There are a few limitations of our current study that we would like to discuss. First, several of the relationships between neuroanatomical and clinical measures and the suggestion that atrophy and lesions may reflect distinct pathways to depression are based on statistical approaches/ correlations between these measures. While we acknowledge that correlations alone do not prove causality, our observations are consistent with established structure:function relationships in the brain. Second, this is a cross-sectional study with samples recruited largely from a University based geriatric clinic. The extent to which this sample and consequently our findings reflect community based patients with depression is therefore unclear. Also, the possibility that a subgroup of our patients with smaller frontal lobe volumes are in the preclinical phase of a degenerative disorder cannot be entirely be ruled out. Despite these caveats, our observations are the first to fully integrate quantitative neuroanatomical measures with relevant medical indices and offer an early glimpse into plausible mechanisms and pathways that contribute to mood disturbances. Our findings, albeit cross-sectional in nature, represent an important first step in elucidating the complex pathways and circuits that underlie clinical depression in the elderly. 
In summary, our data indicate that atrophy and high intensity lesions represent complementary, and somewhat independent paths to late-life MDD. While these two phenomena may overlap in certain clinical situations and at a cellular level, the primary origins of the two pathways in MDD, i.e. neurodegeneration and high intensity lesions, are likely to be different, each of them arising as a consequence of fundamentally different processes. Disruption of critical corticocortical and subcorticocortical circuits by atrophy, ischemia, or a combination of mechanisms could provide a neurobiological substrate that increases vulnerability to overt clinical disturbances. When the neurobiological burden crosses a threshold, either independently or in the presence of other acute medical or psychosocial challenges, the end point may be a serious clinical disturbance like MDD. These observations have broad pathophysiological implications for mood disorders in general as they conceptually narrow the differences between medical and psychiatric illness and illustrate the complementary nature of biological mechanisms in the etiology of affective disorders.

\section{ACKNOWLEDGMENTS}

Supported by grants MH 55115 (to Dr Kumar) and MH 52129 (to Clinical Research Center) from NIMH. Presented in part at the 28th Annual Meeting of the Society for Neuroscience, Los Angeles, 1998.

\section{APPENDIX}

In Figure 2A, the temporal lobe region is drawn onto a 5 -mm axial T2 image. The region delineated is representative of temporal lobe drawings for all slices inferior to the one shown. In Figure 2B, the frontal and temporal lobe regions are drawn on a slice located $1 \mathrm{~cm}$ superior to that shown in A The line used to delineate the posterior temporal border is depicted with dashes from the anteriormost tip of the contralateral cerebral peduncle to the anteromedialmost tip of the cerebellum. The frontal and temporal lobe regions drawn onto slices located $1 \mathrm{~cm}$ and $2 \mathrm{~cm}$ superior to that shown in B are depicted in $C$ and $D$, respectively. All frontal re-

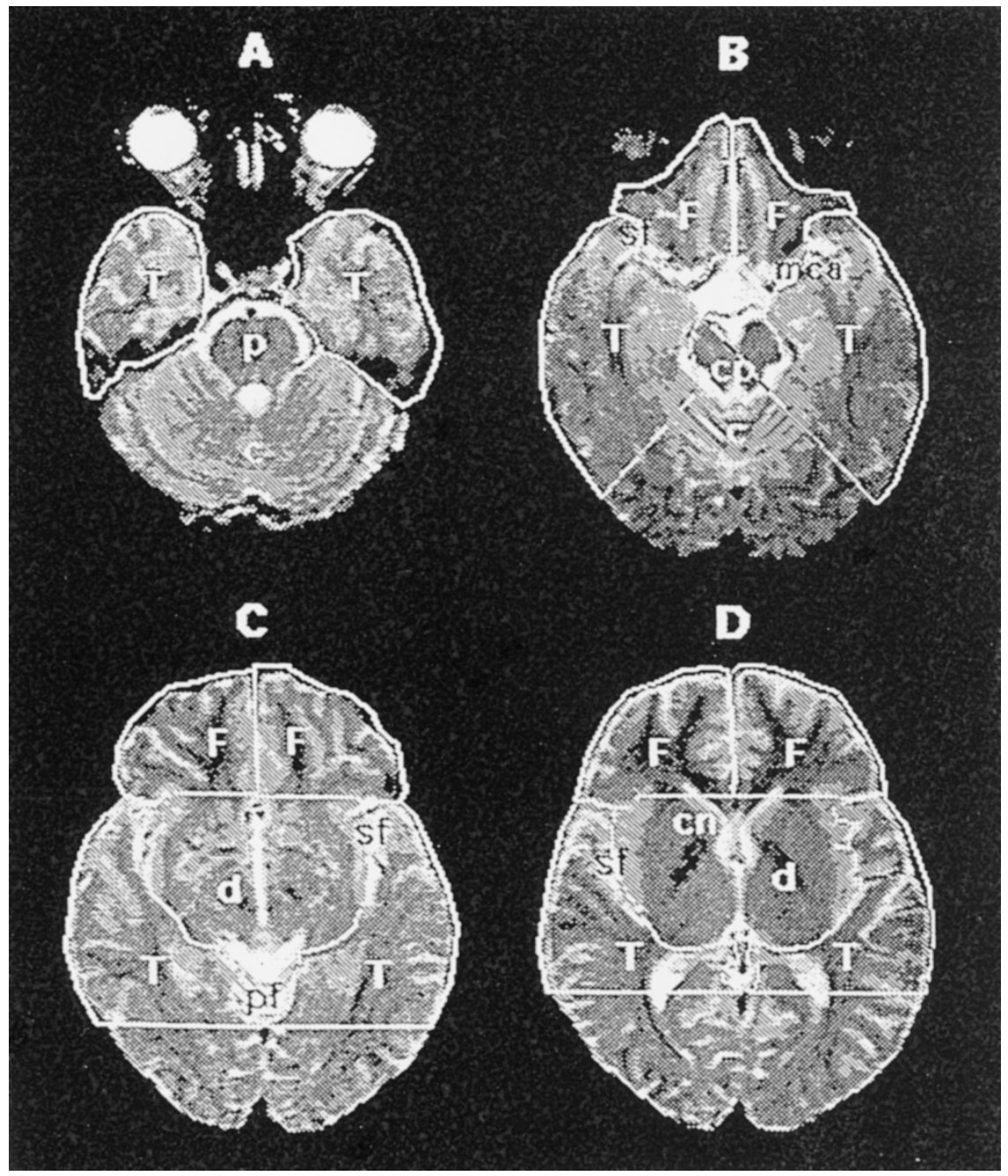

Figure 2. The boundaries of the frontal and temporal lobes from the inferior to the superior slices of the brain. $C$, cerebellum; $c n$, caudate nucleus; $c p$, cerebral peduncle; $F$, frontal region; $d$, diencephalon; if, interhemispheric fissure; $m c a$, middle cerebral artery; $p$, pons; $s f$, sylvian fissure; $T$, temporal region. 
gions drawn on slices superior to the one shown in $\mathrm{D}$ used the same posterior boundary. It should be noted that all regions drawn encompass tissue to be further segmented into brain and CSF volumes.

Neuroradiologic evaluation of MRI's. Prior to regional measurement, brains were realigned in three dimensions using the software package PETVIEW 1.1 and resliced along the AC-PC axis to standardize for differences in head tilt during image acquisition. Resliced images were then imported into another computer software package (Kohn et al., 1991) modified to accommodate regional analysis. The borders of the frontal and temporal lobes were drawn by investigators working with a neuroradiologist using standardized boundaries.

In the inferiormost slices, the temporal lobe did not share common lateral or anterior borders with other structures and was easily outlined. The posteromedial temporal lobe border was formed by the pons and cerebellum (Figure 2A). At the level of the midbrain, borders of the frontal lobe were drawn along the interhemispheric fissure and followed the middle cerebral artery through the suprasellar cistern (Figure 2B). The temporal lobe was separated from adjacent frontal regions by the Sylvian fissure within which runs the middle cerebral artery. The amygdala and hippocampus were included within the temporal lobe, and the midbrain structures were excluded (Figure 2B). The posterior temporal lobe was delineated by a line extending from the anteriormost tip of the contralateral cerebral peduncle to the anteromedialmost tip of the cerebellum (the dashed line in Figure 2B).

Above the level of the mammillary bodies, the posterior border of the frontal lobe was delineated by a horizontal line that extended from the anteromedial-most aspect of the Sylvian fissure to midline (Figure 2C). The medial borders of the temporal lobe were the Sylvian fissure and structures of the diencephalon (Figure 2C, D). A horizontal line, extending from the posteriormost tip of the posterior fossa to the lateral cortical perimeter, delineated the posterior temporal lobe (Figure 2C). The posterior border for the remaining superior slices containing frontal lobe was delineated in the slice immediately inferior to the crossing of the splenium of the corpus callosum (Figure 2D). A line defined by the anteriormost aspect of the caudate was drawn from the midline to the Sylvian fissure. This slice was also the most superior location at which temporal lobe borders were drawn. Adapted from Cowell et al, J Neuroscience, 1994

\section{REFERENCES}

Alexopolous G, Young RC, Meyers BS (1988): Late-onset depression. Psychiatry Clin North Am 11:101-105
Alexopolous GS, Meyers BS, Young RC, Kakuma T, Silbersweig D, Charlson M (1997): Clinically defined vascular depression. Am J Psychiatry 154:562-565

American Heart Association (1990): Stroke risk factor prediction chart. Dallas, American Heart Association

American Psychiatric Association (1994): Diagnostic and statistical manual of mental disorders, 4th ed. Washington, DC, American Psychiatric Press

Baxter LR, Phelps ME, Mazziotta JC, Guze BH, Schwartz JM, Selin CE (1987): Local cerebral glucose metabolic rates in obsessive-compulsive disorder. Arch Gen Psychiatry 44:211-218

Beck DA, Koenig HG (1996): Minor Depression: a review of the Literature. International J Psychiatry in Medicine 26:177-209

Berkman LF, Berkman CS, Kasl S, Freeman DH, Leo L, Ostfeld AM, Cornonti-Huntley J, Brody JA (1986): Depressive symptoms in relation to physical health and functioning in the elderly. American Journal of Epidemiology 124:372-388

Blazer DG, Hughes DC, George LK (1987): The epidemiology of depression in an elderly community population. Gerontologist 27:281-287

Blazer DG (1989): Depression in the elderly. N Eng J Med 320:164-166

Blazer DG (1994): Dysthymia in community and clinical samples (editorial). Am J Psychiatry 151:1567-1569

Boyko OB, Alston SR, Fuller GN (1994): Utility of postmortem magnetic resonance imaging in clinical neuropathology. Arch Pathol Lab Med 118:219-225

Caine ED, Lyness JM, King DA (1993): Reconsidering depression in the elderly. Am J Geriatr Psychiatry 1:4-20

Coffey CE, Figiel GS, Djang WT (1990): Subcortical hyperintensity on magnetic resonance imaging: a comparison of normal and depressed elderly subjects. Am J Psychiatry 147:187-189

Coffey CE, Wilkinson WE, Weiner ARD (1993): Quantitative cerebral anatomy in depression: a controlled magnetic resonance imaging study. Arch Gen Psychiatry 50:7-16

Cowell PC, Turetsky BI, Gur RC, Grossman RI, Shtasel DL, Gur RE (1994): Sex differences in aging of the human frontal and temporal lobes. J Neurosci 14:4748-4755

DeReuck J, Crevits L, De Coster W (1980): Pathogenesis of Biswanger's chronic subcortical encephalopathy. Neurology 30:920-928

Drayer B (1988): Imaging of the aging brain (normal findings and pathologic conditions). Radiology 166:785-806

Duman RS, Heninger GR, Nestler EJ (1997): A molecular and cellular theory of depression. Arch Gen Psychiatry 54:597-606

Fisher MC (1989): Biswanger's encephalopathy: a review. J Neurol 236:65-79

Folstein MF, Folstein SE, McHugh PR (1975): Mini-mental state: a practical method for grading the cognitive state of patients for the clinician. J Psychiatry Res 12:189-198

Frasure-Smith N, Lesperance F, Talajic M (1995): Depression and 18-month prognosis after myocardial infarction. Circulation 91:999-1005

Fuster JM (1989): The prefrontal cortex: anatomy, physiol- 
ogy, and neuropsychology of the frontal lobe. New York, Raven Press.

Fuster JM (1996): Frontal Lobe Syndromes. In Fogel BS, Schiffer RB, Rao SM, (eds), Neuropsychiatry. Baltimore, Williams and Wilkins, pp 407-413

Gierz M, Jeste DV (1993): Physical comorbidity in elderly schizophrenic and depressed patients. American Journal of Geriatric Psychiatry 1:165-170

Glassman AH, Shapiro PA (1998): Depression in the course of coronary artery disease. American Journal of Psychiatry 155:4-11

Greenwald BS, Kramer-Ginsberg E, Krishnan KRR (1996): MRI signal hyperintensities in geriatric depression. Am J Psychiatry 153:1212-1215

Hamilton M (1967): Development of a rating scale for primary depressive illness. British Journal of Social and Clinical Psychology 6:278-296

Hastak SM, Hachinski VC (1992): Multi-infarct dementia: an expanding concept. In Barnett HJM, Mohr JP, Stein BM, Yatsu FM, eds. Stroke: pathophysiology, diagnosis, and management, 2nd ed. New York, Churchill-Livingstone, pp. 793-803

Huang K, Wu L, Luo Y (1985): Biswanger's disease: progressive subcortical encephalopathy or multi-infarct dementia? Can J Neurol Sci 12:88-94

Jeste DV, Lohr JB, Goodwin FK (1988): Neuroanatomical studies of major affective disorders: a review and suggestions for further research. Br J Psychiatry 153:444-459

Katz IR, Streim J, Parmalee P (1994): Psychiatric-medical comorbidity: implications for health services delivery and for research on depression (editorial). Biol Psychiatry 36:141-145

Katz IR (1996): On the inseparability of mental and physical health in aged persons: lessons from depression and medical comorbidity. Am J Geriatr Psychiatry 4:1-16

Koenig HG, O'Connor CM, Guarisco SA (1993): Depressive disorder in older medical inpatients on general medical and cardiology services at a university teaching hospital. Am J Ger Psychiatry 3:197-210

Kohn MI, Tanna NK, Herman GT (1991): Analysis of brain and cerebrospinal fluid volumes with MR imaging. Radiology 178:115-122

Krishnan KRR, Goli V, Ellinwood EH (1988): Leukoencephalopathy in patients diagnosed as major depressive. Biol Psychiatry 23:519-522

Krishnan KRR (1993): Neuroanatomical substrates of depression in late life. J Geriatric Psychiatry Neurol 6:39-58

Krishnan KRR, Hays JC, Blazer DG (1997): MRI-defined vascular depression. Am J Psychiatry 154:497-501

Kumar A, Gottlieb G (1993): Frontotemporal dementias: a new clinical syndrome? The Am J Ger Psychiatry 1:95108

Kumar A, Newberg A, Alavi A (1993): Regional cerebral glucose metabolism in late-life depression and Alzheimer's disease. Proc Natl Acad Sci U S A 90:7019-7023

Kumar A, Newberg A, Alavi A (1994): MRI volumetric studies in Alzheimer's disease: relationship to clinical and neuropsychological variables. Am J Ger Psychiatry 2:21-31
Kumar A, Miller D, Ewbank D, Yousem D, Newberg A, Samuels S, Cowell P, Gottlieb G (1997a): Quantitative neuroanatomic measures and comorbid medical illness in late life depression. Am J Ger Psychiatry 5:15-25

Kumar A, Schweizer E, Zhisong J, Miller, D, Bilker, W, Swan L et al (1997b): Neuroanatomical substrates of late-life minor depression. Archives of Neurology 54:613-617

Kumar A, Zhisong J, Bilker W, Udupa J, Gottlieb G (1998): Late-onset minor and major depression: early evidence of common neuroanatomical substrates detected using MRI. Proc Natl Acad Sci 95:7654-7658

Lacro JP, Jeste DV (1994): Physical comorbidity and polypharmacy in older psychiatric patients. Biol Psychiatry 36:146-152

Lesser IM, Mena I, Boone KB (1994): Reduction of cerebral blood flow in older depressed patients. Arch Gen Psychiatry 51:677-686

Linn BS, Linn MW, Gural L (1968): Cumulative illness rating scale. J Am Geriatr Soc 16:622-626

Loizou LA, Kendall BE, Marshall J (1981): Subcortical arteriosclerotic encephalopathy: a clinical and radiologic investigation. J Neurol Neurosurg Psychiatry 44:294-304

Longstreth WT, Manolio TA, Arnold A, Burke GL, Bryan N, Jungreis CA, Enright DL, O'Leary D, Fried L (1996): Clinical correlates of white matter findings on cranial magnetic resonance imaging of 3301 elderly people: the cardiovascular health study. Stroke 27:1274-1282

Lyness JM, Caine ED, Cox C (1998): Cerebrovascular risk factors and later-life major depression: testing a small-vessel brain disease model. Am J Ger Psychiatry 6:5-13

Mishkin M (1964): Preservation of central sets after frontal lesions in monkeys. In Warren JM, Akert K, editors. The frontal granular cortex and behavior. New York, McGraw-Hill, pp 219-241

Morris P, Rapoport SI (1990): Neuroimaging and affective disorders in late life: a review. Can J Psychiatry 35:3473549

NIH Consensus Development Panel on Depression in Late Life: diagnosis and treatment of depression in late life (1992): JAMA 268:1018-1024

Oxman TE, Barrett JE, Barrett J, Gerber P (1987): Psychiatric symptoms in the elderly in a primary care practice. Gen Hosp Psychiatry 9:167-173

Parmalee PA, Katz IR, Lawton MP (1989): Depression in institutionalized aged: assessment and prevalence. J Gerontol 44:M22-M29

Rabins PV, Harvis K, Koven S (1985): High fatality rates of late-life depression associated with cardiovascular disease. J Affective Disorders 9:165-167

Rabins PV, Pearlson GD, Aylward E (1991): Cortical magnetic resonance imaging changes in elderly inpatients with major depression. Am J Psychiatry 148:617-620

Robinson RG, Kubos KL, Starr LB, Rao K, Price TR (1984): Mood disorders in stroke patients. Brain 107:81-93

Rodin G, Voshart K (1986): Depression in the medically ill: an overview. Am J Psychiatry 143:696-705

Ruegg RG, Zisook S, Swerdlow NR (1988): Depression in the aged: an overview. Psychiatry Clin North Am 11:83-99

Sackheim HA, Prohovnik I, Moeller R (1993): Regional cere- 
bral blood flow in mood disorders, II: comparison of major depression and Alzheimer's disease. J Nucl Med 34:1090-1101

Sapolsky RM, Pulsinelli WA (1985): Glucocorticoids potentiate ischemic injury to neurons: therapeutic implications. Science 229:1397-1400

Sapolsky RM, Krey LC, McEwen BS (1986): The neuroendocrinology of stress and aging: the glucocorticoid cascade hypothesis. Endocrine Reviews 7:284-301

Sapolsky RM, Uno H, Rebert CS (1990): Hippocampal damage associated with prolonged glucocorticoid exposure in primates. J Neurosci 10:2897-2902

Schmidt R, Fazekas F, Offenbacher H (1991): Magnetic resonance imaging white matter lesions and cognitive impairment in hypertensive individuals. Arch Neurol 48:417-420

Seigel DG, Greenhouse SW (1973): Multiple relative risk function in case-control studies. Am J Epidemiology 97:324-331

Sheline YI, Wang PW, Gado MH, Csernansky JG, Vannier MW (1996): Hippocampal atrophy in recurrent major depression. Proc Natl Acad Sci 93:3908-3913

Sherbourne CD, Wells KB, Hays RD, Rogers W, Burnam MA, Judd LL (1994): Subthreshold depression and depressive disorder: clinical characteristics of general medical and mental health specialty outpatients. Am J Psychiatry 151:1777-1784
Stuss DT, Benson DF (1984): Neuropsychological Studies of the Frontal Lobes. Psychological Bulletin 95:3-28

Udupa JK (1994): Multidimensional digital boundaries, CVGIP. Graphical Models and Image Processing 54: 311-323

Udupa JK, Odhner S, Samarasekera R, Goncalves RJ, Iyer K, Venugopal K, Furuie S (1994a): 3DVIEWNIX: an open, transportable, multidimensional, multimodality, multiparametric imaging software system. SPIE Proc. 2164: 58-73

Udupa JK, Samarasekera S (1996): Fuzzy connectedness and object definition: theory, algorithms, and applications in image segmentation. Graphical Models and Image Processing 58:246-261

Weinberger DR, Berman KF, Suddath R, Torrey EF (1992): Evidence of dysfunction of prefrontal-limbit network in schizophrenia: a magnetic resonance imaging and regional cerebral blood flow study of discordant monozygotic twins. Am J Psychiatry 149:890-897

Wolf PA, D'Agostino RB, Belanger AJ (1991): Probability of stroke: a risk profile from the Framingham study. Stroke 22:312-318

Ylikoski A, Erkinjuntti T, Raininko R, Sarna S, Sulkava R, Tilvis R (1995): White matter hyperintensities on MRI in the neurologically nondiseased elderly. Analysis of cohorts of consecutive subjects aged 55 to 85 years living at home. Stroke 26:1171-1177 